\title{
A Case Report and Review of Thymic Carcinoma with Adenoid Cystic Carcinoma like Features
}

\author{
Boyou Zhang*1 and Qing Geng ${ }^{2}$ \\ ${ }^{1}$ Department of Emergency medical department, China \\ ${ }^{2}$ Department of Thoracic Surgery, Wuhan University, China
}

Received: April 19, 2018; Published: 蹧 April 26, 2018

*Corresponding author: Boyou Zhang, Department of Emergency medical department, Jishuitan Hospital, Beijing 100035, China

\section{Introduction}

Adenoid cystic carcinoma (ACC) is also called cylindroma, which is considered as a kind of low-grade malignant tumor and often occurs in head and neck salivary gland tissue. Most of them happened in submandibular gland and minor salivary glands while rarely happened in thymus. There are only 7 cases had already reported before all around the world. Our case is diagnosed as thymic carcinoma with adenoid cystic carcinomalike features by pathology after surgery; it is different with other cases because he had accepted a surgery six years ago because of the mediastinal mass and pleural effusion. Because of the symptom of chest tightness, he came to see doctor again and finally find there was a mediastinal mass and accepted the treatment of surgery. The medical history of our case maybe provides some inspiration of the pathogenic of thymic carcinoma with adenoid cystic carcinoma like features.

\section{Information and history}

A 60 years old male came to our hospital because of chest tightness in 2009/10. The enhanced chest CT showed "A large mass measuring $9.2 * 10.1 \mathrm{~cm}^{2}$ in the right side of the mediastinal which the average CT value is $9 \mathrm{Hu}$. The border with the superior vena cava and the right atrial and mediastinum is less clear. The right side of the chest is hydropneumothorax, Hydropericardium. The ultrasonic showed there is a mass of mixed echo in the right chest which had strong echo light and liquid visible mass dark space inside it. The test of pleural effusion by pleural puncture showed that was hematodes exudates without cancer cells. He accepted a surgery to resection of the mass by posterolateral thoracotomy, the pathology showed "Mostly considered as hematoma with organization according to clinical”. After the surgery ,he recovered and discharged from our hospital.
It is said by himself that there was found a mediastinal mass in his chest by CT in the year of 2010(the imaging data has been lost), and he taking chinese traditional medicein(herbal medicine) for tow month. After that he reexamination a chest CT showed the mediastinal mass was disappeared so he didn't have the follow-up according to the doctor's advice. 2014/10/13, he had a chest CT examination showed there is a $15^{*} 13 \mathrm{~cm}^{2}$ cystic with solid abnormal density shadow in the right front mediastium; Mediastinal lymph node is enlarged; Density of soft tissue in chest wall which is considered as metastatic. He reexamined a chest CT in 2015/1/4 but did not show significant change. Finally, he came to hospital again because of the intermittent chest pain. Complete the checks after hospitalization, lung function show" FEV1 : 3.94L, FEV1/FVC : $79 \%$ ", the enhanced chest CT showed "there is a $15.2 * 11.6 \mathrm{~cm}^{2}$ cystic with solid abnormal density shadow in the right front mediastium, the border is smooth and the density inside is uniform, the CT value is $18 \mathrm{Hu}$; Right pulmonary atelectasis, the chest wall and diagram pars maybe have already metastasis tumor".

a. Anamnesis: The patient with high blood pressure for more than 20 years, he use reserpin and Chinese traditional medicine to ctrl his high blood press by himself, but the effect is poor. His blood pressure was stabled for able $108 / 90 \mathrm{mmHg}$ for all years. Smoking 20 years and quit it for 23 years. We asked his medical history repeatedly, he denied the history of trauma and hemorrhagic disease.

b. Preoperative diagnosis: anterior mediastinum space occupying lesion, malignant tumor is the most possible. The chest wall maybe already metastasized. We suggested him to accept PET/CT to make the tumor's metabolic strength clear and conform the primary focal; Or accept thoracentesis by fine 
needle to definite the pathological diagnosis. The patient and his family refused it and screamed for an operation.

So the surgery was planned. The patient was placed in the supine position under general anesthesia. Via median sternotomy. We saw the tumor was located in right anterior mediastinum, $12 * 12 * 10 \mathrm{~cm}^{3}$, it was dense adhesion with mediastinal pleura, pericardium, diaphragm and right lung. The tumor is invading and partly wrapped around superior vena cava. Cut out part of the tumor tissue and sent to the examination of fast frozen section and the result was benign tumor. Then we completed removal of the tumor and cut one right rib and the tumor on the chest wall, the fast frozen section also report "benign tumor". The tumor was solid and multicystic hemorrhagic with many sediment sample material. The diagnosis of the frozen section considered that most possible is encapsulated empyema. So we use polyninylpyrrolidone and normal saline to rinse the chest repeatedly and the closed thoracic conventionally.

The result of postoperative pathological: thymic carcinoma with adenoid cystic carcinomalike features with chest wall invasion, the tissue of cystiform is hyperplasia of fibrous tissue and stale hemorrhage. The result of immunohistochemical : $\mathrm{CK}(+)$, $\operatorname{EMA}(-), \operatorname{CK}(\mathrm{L})(+), \mathrm{E}-\mathrm{Ca}(++), \mathrm{ER}(+), \operatorname{PR}(-), \mathrm{Ki67}(10 \%), \mathrm{CK} 8 / 18(+)$ CD56 Focal(+), NSE(-), Syn(-), CgA(-).After the surgery, we checked the patient's oral cavity, head and neck region but not found and obvious abnormal. 6 month pasted and we have not found any indication of tumor recurrence. He is still in follow up now.

\section{Discussion}

Adenoid cystic carcinoma is salivary gland tumor, but can still be reported in breast [1], lung [2], esophageal [3], postate [4]. Thymic carcinoma with adenoid cystic carcinoma like features is very rare. Only 7 cases have already be reported all around the world before while all cases is not in China.

Thymic carcinomas are defined as thymic epithelial neoplasmas, which are classified into 10 different histological types according to the 2015 update of the WHO classification, i.e.[5]
a. squamous-cell carcinoma
b. basal cell carcinoma
c. mucoepidermoid carcinoma
d. lymphoepithelioma-like carcinoma
e. sarcomatoid carcimoa
f. clear cell carcinoma
g. adenocarcinoma
h. NUT carcinoma
i. unclassified carcinoma
j. other rare thymic carcinoma.

Within the group of adenocarcinoma, four histologic subtypes are known: papillary adenocarcinoma; thymic carcinoma with adenoid cystic carcinomalikefeatures; mucinous adenocarcinoma; adenocarcinoma, non specifically. Compared with the 2004 update of the WHO classification, the name of adenoid cystic carcinoma is changed to thymic carcinoma with adenoid cystic carcinomalike features. It lack the real features of adenoid cystic carcinoma in the character of immunohistochemical because it is generate from the thymus (Table 1) [6-8].

Table 1: The character of the cases of thymic carcinoma with adenoid cystic carcinomalike features which are reported so far.

\begin{tabular}{|c|c|c|c|c|c|c|}
\hline author & time & age/sex & $\operatorname{size}(\mathrm{cm})$ & Distant metastasis & therapies & Follow up \\
\hline \multirow[t]{4}{*}{ Di Tommaso } & 2007 & $65 / \mathrm{male}$ & 5 & no & surgery & lost \\
\hline & & 63/female & $2.5 * 2$ & no & surgery & lost \\
\hline & & $69 /$ male & $13 * 10$ & no & surgery /radiotherapy & Died of other causes; $62 \mathrm{mo}$ \\
\hline & & $77 /$ male & $13 * 10$ & no & surgery & Alive without disease $; 2 \mathrm{mo}$ \\
\hline Coulibaly & 2008 & $37 /$ female & Large & no & surgery /chemotherapy/radiotherapy & Alive without disease; $7 \mathrm{mo}$ \\
\hline Banki & 2010 & $65 /$ female & $14 * 8.8$ & no & surgery & Alive without disease; $30 \mathrm{mo}$ \\
\hline Ryu Kanzaki & 2011 & $66 /$ male & $4.9 * 4.4$ & yes & surgery & Alive with disease; $12 \mathrm{mo}$ \\
\hline This example & 2015 & $66 /$ male & $15^{*} 11$ & no & surgery & Alive without disease; $6 \mathrm{mo}$ \\
\hline average & & 63.5 & $9.6 * 7.3$ & & & \\
\hline
\end{tabular}

\section{The Character of Clinical}

All of the clinical character of the cases which have already been reported is summarized above (chart-1). Thymic carcinoma with adenoid cystic carcinoma like features is very rare, most of them are occurred in elderly and few distant metastases. But it can show the character of aggressive growth and directly invasive chest wall; pericardium and other adjacent tissue. The initial symptoms of the patients are fever; cough; dyspnea; chest pain; the sense of suppression in the chest and the loss of weight [9]. thymic carcinoma with adenoid cystic carcinoma like features is generated in the tissue of thymus which located in the mediastinum, and the size of the most tumor is large. The severity of the clinical symptoms is uncorrelated with the size of the tumor.

Although the size of the tumor is large, the symptoms of patients is not severely even cannot let them go to see doctor. The clinical symptoms is formed because of the oppress by the tumor, there is 
no obviously symptoms in early stage, with the growth of tumor , the patient will appear the atypical symptoms such as cough; fever; dyspnea; chest pain and so on. Because thymic carcinoma with adenoid cystic carcinomalike features is a low grade malignancy, all cases had the features of gradual onset and grow progress. So only one case that had already metastases to rib was reported that is different whit the character of adenoid cystic carcinoma in salivary. According to the Masaoka stage of thymus tumor, our case is in stage $\beta$.

\section{The Character of Pathology}

The macroscopic view of the tumor: Most of them are consist of huge gritty and firm cystic solid mass with smooth border. Some of them have complete capsule. The cross-section showed multicystic, necrotic tissue mixed with fibrotic, yellow tissue nodules. Some of them can be observed the papillary hyperplasia and plenty of hemorrhagic effusion.

a. The microscopic view of the tumor: The character of our case is similar as salivary adenoid cystic carcinoma. The cystic and cribriform texture neoplastic nest is consist of basoloid cell carcinoma ,and bloody fluid or granular basophilic myxoid stroma full of the cyst. The microscopic view of the thymic carcinoma with adenoid cystic carcinomalike features can be classified as three types. If the tumor cell is undifferentiated, it will show the solid structure with irregular funicular or dense array by basaloid cells(solid type). If the tumor cell is differentiation to the glandular epithelium, it will show the gland tubular structure consist of the inner layer of columnar epithelium and the outside of the myoepithelial cells with homogeneous eosinophilic mucin full of the cavity of the tubule(tubular type). If the tumor cell is differentiation to the myoepithelial cell, it will show the cribriform structure with homogeneous basophilic cell matrix(cribriform type)[10]. The classify of thymic carcinoma with ACC like features is closely connected with the prognosis, so we infer that the prognosis of solid type is the worst while the prognosis of tubular type and the cribriform type is more better.

b. Immuno histochemistry: $\mathrm{CK}$ and $\mathrm{CKL}$ all showed positive in our case, they were marked at the epithelium which consist of cribriform structure. E-Ca(++) prompted the tumor has a certain invasive in accordance with the invasion of chest wall. Ki67(10\%) prompted the active proliferation. CD56(focal + ) is the new character that has not be reported before ER(+) prompted this case may be sensitive to endocrine therapy, but there isn't any evidence of evidence-based medicine support it so the clinical significance needs to be confirmed.

Summarize other documents, we can get the following conclusion:

i. These marks is contribute to the diagnosis of thymic carcinoma with adenoid cystic carcinomalike features if they are positive: CollagenIV, laminin, P63, CK34betaE12, Ki67(1$10 \%)$

ii. These marks is contribute to the diagnosis of thymic carcinoma with adenoid cystic carcinomalike features if they are negative: Syn, NSE, CD117, CgA.

The molecular marks research of this disease has not yet been carried out. There are reports that suggested MYB-NFIB fusion gene, NF- $\mathrm{kB}, \mathrm{MMP}-2$, survivin is related to adenoid cystic carcinoma [11], and it is helpful to the molecular marks research of thymic carcinoma with adenoid cystic carcinomalike features.

\section{The Differential Diagnosis}

PET/CT has great significance to this disease in differential diagnosis, it can estimate whether the mass is primary tumor or metastases. The report suggested the tumor of this disease show high value of SUV max in PET/CT while its metastases can also show unusually high value of SUV max [9]. The retrospective analysis show that have a examination of PET /CT before surgery is correct and meaningful. In the primary thymic carcinoma, this disease should be distinguished with basaloma. In morphology, most of the tumor cell of basaloma is cubic cells with small and deep dyeing nucleus which array form lobulated or funicular $[12,13]$. The results of Immuno histochemistry can confirm that our case can be diagnosed as thymic carcinoma with adenoid cystic carcinoma like features.

\section{The treatment and prognosis}

Surgery is given priority to this disease at present and radiation therapy can according to the situation. The effect of chemotherapy for this disease is poor and if take chemotherapy as a routine treatment is still controversial. Some professional insist of that adenoid cyst carcinoma cannot get enough benefit from chemotherapy, so we should treat chemotherapy as the last treatment [14]. We believe that A case report and review of thymic carcinoma with adenoid cystic carcinoma like features is a low level malignant tumor so the it should have better prognosis after standardized treatment. But we still should be carefully evaluated before surgery and if we suspect the diagnosis as thymic tumor with ACC like carcinoma or huge mediastinal mass, PET/CT will be suggested to confirm the quality of the mass and whether there is metastases or not.

Locally metastasis and the chest wall invasion should not be the absolute contraindications of surgery because as a low level malignant tumor, if we resection the tumor and metastases completely and take some radiation therapy appropriately, nice quality of life will get. In our case a recurrence is appear after the surgery in 2009 , but he said itself that the mediastinal mass was once disappeared after the treatment of Chinese traditional medicine. So we guess that Chinese traditional medicine may effective for this disease. 


\section{The discussion of pathogenesis}

A case report and review of thymic carcinoma with adenoid cystic Carcinoma like features is a kind of thymic carcinoma, it is generated from thymic epithelial cells. If the tumor cell is differentiation to the myoepithelial cell, it will show the cribriform structure with homogeneous basophilic cell matrix. The medical history of our case is so specially that he found the mediastinal mass six years ago and experienced the surgery. The pathological report at the time of 2009 showed "hematoma with organization". Organization means the absorption process by new granulation tissue if necrotic tissue, thrombus, pus or foreign body cannot be dissolved or absorbed or separated or discharged. The granulation tissue contains abundant myocyte and some of them will differentiate to myofibroblast after the stimulate of cytokines.

And these cytokines that can induce differentiation may induce the differentiate thymic epithelial cell to myoepithelium and finally generate to the type of cribriform of thymic carcinoma with adenoid cystic carcinomalike features is a kind of thymic carcinoma. This maybe one of the triggers of this disease although still need to establish by experiments. According to the inference above, we consider coagulate hemothorax and mediastinal hematoram may result thymic carcinoma with adenoid cystic carcinoma like features. In order to avoid the remained thymus tissue be stimulated to differentiate to thymic carcinoma, in the surgery of mediastinal hematoma, thymectomy completely is necessary and fat tissue of mediastinal should be resection completely too.

\section{References}

1. Azoulay S, Laé M, Fréneaux P, Merle S, Al Ghuzlan A, et al. (2006) KIT is highly expressed in adenoid cystic carcinoma of the breast, a basallike carcinoma associated with a favorable outcome. Modern Pathology 18(12): 1623-1631.

2. Elizabeth A, Thomas L, Harrell JH (2004) Tracheobronchial adenoid cystic carcinoma: a clinicopathologic study of 14 cases. Chest $125(3)$ 1160-1165.
3. Jiang Wenyang, Fu Xiangning, Fu Shengling (2012) Report of one case of adenoid cystic carcinoma of the esophagus. Journal of Huazhong University of Science and Technology: Medical Journal 1: 115-116.

4. Mckenney J K, Amin M B, Srigley J R, Jimenez RE, Ro JY, et al. (2004) Basal cell proliferations of the prostate other than usual basal cell hyperplasia: a clinicopathologic study of 23 cases, including four carcinomas, with a proposed classification. American Journal of Surgical Pathology 28(10): 1289-1298.

5. (2015)Thymic neoplasm histological classification, WHO, USA

6. Di Tommaso L, Kuhn E, Kurrer M, Zettl A, Marx A, et al. (2007) Thymic tumor with adenoid cystic carcinoma like features: a clinicopathologic study of 4 cases . American Journal of Surgical Pathology 31(8): 11611167.

7. Coulibaly B, Marcy M, Fernandez C, PA Thomas, Roger G (2008) Primary thymic adenoid cystic carcinoma mimicking a teratoma: a case report.

8. Banki F, Khalil K, Kott M M (2010) Adenoid cystic carcinoma of the thymus gland: a rare tumor. The Annals of thoracic surgery $90(4)$ : e56-e58.

9. Kanzaki R, Ikeda N, Okura E, kitahara N, Okimura A, et al. ( 2012) Thymic carcinoma with adenoid cystic carcinomalike features with distant metastases. Annals of Thoracic \& Cardiovascular Surgery Official Journal of the Association of Thoracic \& Cardiovascular Surgeons of Asia 18(6): 544-547.

10. Fordice J, Kershaw C, El Naggar A, Goepfert H (1999) Adenoid cystic carcinoma of the head and neck: predictors of mobidity and mortality. Arch Otolaryngol Head Neck Surg 125: 149-152.

11. Zhang Li, Zhang Xiaozhi (2015) Recent advances in molecular markers of adenoid cystic carcinoma. Modern Oncology Medicine 23(5): 726-730.

12. Hernandez Rios P, Fan F (2007) Metastatic thymic carcinoma with hyaline stromal material mimicking adenoid cystic carcinoma. Diagnostic cytopathology 35(4): 225-226.

13. Brown J G, Ubaldo F, Mauro P, Rosai J (2009) Thymic basaloid carcinoma: a clinicopathologic study of 12 cases, with a general discussion of basaloid carcinoma and its relationship with adenoid cystic carcinoma. American Journal of Surgical Pathology 33(8): 1113-1124.

14. Laurie SA, Su YB, Pfister DG (2005) Chemotherapy in the management of metastatic adenoid cystic carcinoma: A systematic review. Journal of Clinical Oncology (16): 520S.
This work is licensed under Creative Commons Attribution 4.0 License

To Submit Your Article Click Here: Submit Article

DOI: 10.32474/OAJOM.2018.01.000117

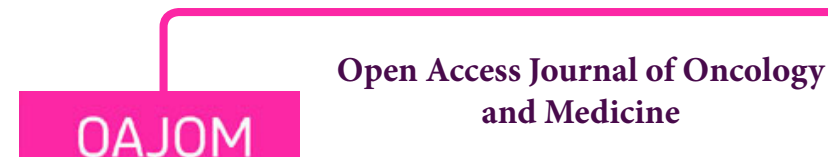

Assets of Publishing with us

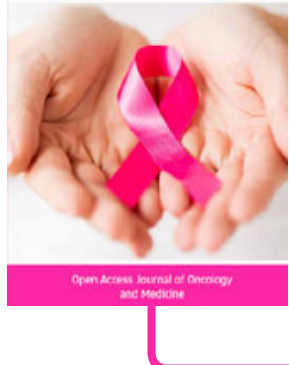

- Global archiving of articles

- Immediate, unrestricted online access

- Rigorous Peer Review Process

- Authors Retain Copyrights

- Unique DOI for all articles 